\title{
Determination of Optimal Supply When Demand Is a Sum of Components
}

\section{Vijayakumar Raman, Venkatesan Thirunavukkarasu, Muthu Chinnathambi}

Department of Statistics, St. Joseph's College, Trichy, Tamil Nadu, India

\section{Email address:}

rvijaystat10@gmail.com (V. Raman), venkateshmaths80@gmail.com (V. Thirunavukkarasu), mailtomuthuinbox@gmail.com (M. Chinnathambi)

\section{To cite this article:}

Vijayakumar Raman, Venkatesan Thirunavukkarasu, Muthu Chinnathambi. Determination of Optimal Supply When Demand Is a Sum of Components. Mathematical Modelling and Applications. Vol. 3, No. 6, 2017, pp. 68-74. doi: 10.11648/j.mma.20170206.13

Received: September 27, 2017; Accepted: November 7, 2017; Published: December 14, 2017

\begin{abstract}
Among the various inventory systems our method is used to find the optimal supply size. To find the optimal supply size taking in to consideration the aspects like inventory holding cost per unit, cost of shortage per unit etc., In many situation the demand taken to be a random variable. The total demand is in turn a sum of three random variables namely (i) demand due to consumers (ii) demand due to the supply of the product to sister concerns or companies. (iii) Demand due to replacement of defective items that are not accepted and hence exchanged for new units Under these assumptions the optimal supply size is derived.
\end{abstract}

Keywords: Demand for the Product, Optimal Supply Size, Sum of Random Variables, Convolution Principle

\section{Introduction}

In inventory control theory, determination of the optimal order size is an important aspect. Similarly there are many situations where the optimal size of the reserve inventory and optimal supply size are to be determined. The optimal supply size is found out taking in to consideration the aspects like inventory holding cost perunit, cost of shortage perunit etc., There are many situations where the demand is taken to be a random variable and hence it has the corresponding probability distribution. Using the different costs involved and the demand size, the optimal quantity of supply is determined.

A detailed account of such models is found in Hansmann (1961). The so called Newsboy problem is one in which the demand is taken to be a random variable and the optimal supply size is determined taking in to consideration the cost of excess stock and the cost of shortages. This basic model has been discussed in Hansmann (1961) Sheik Udumaneet al., (2007) have discussed this model under the assumption that the random variable denoting the demand satisfies the so called Setting the Clock Back to Zero (SCBZ) property due to Raja Rao and Talwalker (1990)

The demand for any product or commodity is usually due to the individual consumers and the quantity they consume or use. But there are some cases or situations where the demand may be due to other factors also and they may influence the quantity demanded. So the total demand may be may be represented as the sum of the demands due to other factors or causes.

In this Chapter two models are discussed. In the case of the first model it is assumed that the demand for the product is the sum of two components namely, (i) The demand due to the purchase by the consumers. (ii) The demand due to the transfer of the product to the sister companies whenever the shortage occurs in those companies. Hence the demand may be represented on the sum of two independent random variables.

An extension of this model to the case of the total demand as the sum of three components is discussed. The total demand is the sum of three variables of random nature. In this Chapter it is assumed that the total demand which is a random variable is the sum of three components of individual demands which are of random character. The total demand is in turn a sum of three random variables namely (i) demand due to consumers (ii) demand due to the supply of the product to sister concerns or companies. (iii) demand due to replacement of defective items that are not accepted and hence exchanged for new units Under these assumptions the optimal supply size is derived. 


\subsection{Assumptions}

(i) The demand for a given product is the sum of two types of demands. (a) consumer demand. (b) demand due to the transfer of goods to the sister companies.

(ii) The supply is instantaneous.

(iii) There are only two costs involved namely the cost of storage and cost of shortages.

\subsection{Notations}

$\mathrm{h}=$ Inventory holding cost

$\mathrm{d}=$ Cost of shortage.

$\mathrm{S}=$ Supply size

$\mathrm{X}=$ The demand for the product which is a random variable, with pdf

$\mathrm{f}(\mathrm{x})$ and $\operatorname{cdf} \mathrm{F}(\mathrm{x})$.

Results

\section{Model I}

It is a well known result that the expected cost of overages and shortages is given by

$$
E(C)=h \int_{0}^{s}(S-X) f(x) d x+d \int_{S}^{\infty}(X-S) f(x) d x
$$

Now since the random variable $\mathrm{X}$ which denotes the total demand is the sum of three types of demands, we have to find the distribution of $\mathrm{X}$ which is the sum of two random variables $\mathrm{X}_{1}, \mathrm{X}_{2}$. For that purpose the convolution principle is used.

$$
E(C)=h \int_{0}^{s}[S-X] f(x) d x+d \int_{s}^{\infty}[X-S] f(x) d x
$$

Now we consider the case where $w_{2}=x_{1}+x_{2}$ $x_{1}=$ demand due to consumer purchase, $x_{2}=$ demand due to transfer to sister companies.

$$
f_{w_{2}}(x)=\int_{0}^{x} f x_{1}(y) f x_{2}(x-y) d y
$$

where $w_{2}=x_{1}+x_{2}$ [by convolution theorem] Let $x_{1} \sim \exp \left(\theta_{1}\right), f x_{1}(y)=\theta_{1} e^{-\theta_{1} y}$

$$
\begin{gathered}
f_{w_{2}}(x)=\int_{0}^{x} \theta_{1} e^{-\theta_{1} y} \theta_{2} e^{-\theta_{2}(x-y)} d y \\
=\int_{0}^{x} \theta_{1} e^{-\theta_{1} y} \theta_{2} e^{-\theta_{2} x} e^{\theta_{2} y} d y \\
=\frac{\theta_{1} \theta_{2}}{-\left(\theta_{1}-\theta_{2}\right)} e^{-\theta_{2} x}\left[e^{-\left(\theta_{1}-\theta_{2}\right) x}-1\right] \\
=\frac{\theta_{1} \theta_{2}}{\left(\theta_{1}-\theta_{2}\right)} e^{-\theta_{2} x}\left[1-e^{-\theta_{1} x} e^{\theta_{2} x}\right] \\
=\frac{\theta_{1} \theta_{2}}{\left(\theta_{1}-\theta_{2}\right)}\left[e^{-\theta_{2} x}-e^{-\theta_{1} x}\right]
\end{gathered}
$$

substituting (2) in (1), we get

$$
\begin{gathered}
\frac{d E(C)}{d s}=h \int_{0}^{s}[S-X] \frac{\theta_{1} \theta_{2}}{\left(\theta_{1}-\theta_{2}\right)}\left[e^{-\theta_{2} x}-e^{-\theta_{1} x}\right] d x \\
+d \int_{s}^{\infty}[X-S] \frac{\theta_{1} \theta_{2}}{\left(\theta_{1}-\theta_{2}\right)}\left[e^{-\theta_{2} x}-e^{-\theta_{1} x}\right] d x=0 \\
=\frac{h \theta_{1} \theta_{2}}{\left(\theta_{1}-\theta_{2}\right)} \int_{0}^{s}[S-X]\left[e^{-\theta_{2} x}-e^{-\theta_{1} x}\right] d x+\frac{d \theta_{1} \theta_{2}}{\left(\theta_{1}-\theta_{2}\right)} \int_{s}^{\infty}[X-S]\left[e^{-\theta_{2} x}-e^{-\theta_{1} x}\right] d x=0 \\
=\left\{\frac{h \theta_{1} \theta_{2}}{\left(\theta_{1}-\theta_{2}\right)}[\int_{0}^{s} \overbrace{[S-X] e^{-\theta_{2} x}}^{\left[S-\int_{0}^{s}\right.} \overbrace{[S-X] e^{-\theta_{1} x}}^{(A)} d x]\right. \\
+\frac{d \theta_{1} \theta_{2}}{\left(\theta_{1}-\theta_{2}\right)}[\int_{s}^{\infty} \overbrace{(X-S] e^{-\theta_{2} x}}^{(B)} d x-\int_{s}^{\infty} \overbrace{[X-S] e^{-\theta_{1} x}}^{(X)} d x]=0
\end{gathered}
$$


By Leibnitz rule

$$
\frac{d}{d x} \int_{\phi(x)}^{\psi(x)} f(x, t) d t=\psi^{1}(x) f[\psi(x), x]-\varphi^{1}(x) f[\varphi(x), x]+\int_{\phi(x)}^{\psi(x)} \frac{d}{d x} f(x, t) d t
$$

$$
\begin{array}{ll} 
& \varphi(s)=0, \varphi^{1}(s)=0, \quad \psi(s)=s, \quad \psi^{1}(s)=1 \\
=1\left[(s-s) e^{-\theta_{2} s}\right]-0[]+\int_{0}^{s} \frac{d}{d s}(S-X) e^{-\theta_{2} x} d x & \text { By Leibnitz rule } \Rightarrow \int_{s}^{\infty}[X-S] e^{-\theta_{2} x} d x
\end{array}
$$

$$
\begin{gathered}
=\frac{1}{-\theta_{2}}\left[e^{-\theta_{2} s}-1\right] \\
\frac{d A}{d s}=\frac{1}{\theta_{2}}\left[1-e^{-\theta_{2} s}\right] \\
\text { (B) } \Rightarrow \int_{0}^{s}[S-X] e^{-\theta_{1} x} d x
\end{gathered}
$$

By Leibnitz rule

$$
\begin{gathered}
\frac{d c}{d s}=-\frac{1}{\theta_{2}}\left[e^{-\theta_{2} s}\right] \\
\text { (D) } \Rightarrow \int_{s}^{\infty}[X-S] e^{-\theta_{1} x} d x
\end{gathered}
$$

By Leibenitz rule

$$
=\frac{1}{\theta_{1}}\left[0-e^{-\theta_{1} s}\right]
$$

By Leibenitz rule

$$
\begin{aligned}
& =\frac{1}{-\theta_{1}}\left[e^{-\theta_{1} s}-1\right] \\
& \frac{d B}{d s}=\frac{1}{\theta_{1}}\left[1-e^{-\theta_{1} s}\right] \\
& =\frac{\theta_{1} \theta_{2}}{\left(\theta_{1}-\theta_{2}\right)}\left[\frac{h}{\theta_{2}}\left[1-e^{-\theta_{2} s}\right]-\frac{h}{\theta_{1}}\left[1-e^{-\theta_{1} s}\right]-\frac{d}{\theta_{2}} e^{-\theta_{2} s}+\frac{d}{\theta_{1}} e^{-\theta_{1} s}\right]=0 \\
& e^{-\theta_{2} s}\left(-\frac{h}{\theta_{2}}-\frac{d}{\theta_{2}}\right)+e^{-\theta_{1} s}\left(\frac{h}{\theta_{1}}+\frac{d}{\theta_{1}}\right)=\frac{h}{\theta_{1}}-\frac{h}{\theta_{2}} \\
& \frac{-e^{-\theta_{2} s}}{\theta_{2}}(h+d)+\frac{e^{-\theta_{1} s}}{\theta_{1}}(h+d)=h\left[\frac{\theta_{2}-\theta_{1}}{\theta_{1} \theta_{2}}\right] \\
& (h+d)\left[\frac{-e^{-\theta_{2} s}}{\theta_{2}}+\frac{e^{-\theta_{1} s}}{\theta_{1}}\right]=h\left[\frac{\theta_{2}-\theta_{1}}{\theta_{1} \theta_{2}}\right] \\
& (h+d)\left[-\theta_{1} e^{-\theta_{2} s}+\theta_{2} e^{-\theta_{1} s}\right]=h\left[\theta_{2}-\theta_{1}\right] \\
& \frac{h}{h+d}=\frac{\left[-\theta_{1} e^{-\theta_{2} s}+\theta_{2} e^{-\theta_{1} s}\right]}{\left[\theta_{2}-\theta_{1}\right]}
\end{aligned}
$$$$
=\frac{h \theta_{1} \theta_{2}}{\left(\theta_{1}-\theta_{2}\right)}\left[\frac{1}{\theta_{2}}\left[1-e^{-\theta_{2} s}\right]-\frac{1}{\theta_{2}}\left[1-e^{-\theta_{1} s}\right]\right]+\frac{d \theta_{1} \theta_{2}}{\left(\theta_{1}-\theta_{2}\right)}\left[-\frac{1}{\theta_{2}}\left[e^{-\theta_{2} s}\right]+\frac{1}{\theta_{1}}\left[e^{-\theta_{1} s}\right]\right]=0
$$

substituting (4), (5), (6) and (7) values in (3)., we get

Hence $\frac{h}{h+d}=\left[\frac{\theta_{2} e^{-\theta_{1} s}-\theta_{1} e^{-\theta_{2} s}}{\theta_{2}-\theta_{1}}\right]$

The optimal $\mathrm{S}$ value is determined by assuming specific values for $\theta_{1}, \theta_{2}$, h and $\mathrm{d}$. 


$$
\begin{aligned}
& \theta_{1}=1.2, \quad \theta_{2}=1.5, \quad h=10, \quad d=50 \\
& \text { LHS }=\frac{10}{10+50} \\
&=\frac{10}{60}
\end{aligned}
$$

LHS $=0.1667$

By giving different values for $\mathrm{S}$, the optimal value can be determined.

$\mathrm{S}=2.4 \mathrm{RHS}=0.1714$

$\mathrm{S}=2.5$ RHS $=0.1547$

Hence $\hat{S}$ lies between 2.4 and 2.5 .
$\mathrm{S}=2.43 \mathrm{RHS}=0.1663$

$S=2.43$

\section{Model II}

In this model it is assumed that $w_{3}=x_{1}+x_{2}+x_{3}$, where $x_{1}=$ consumer demand,

$x_{2}=$ demand due to the sister companies,

$x_{3}=$ demand due to replacement of defective units.

$$
\begin{aligned}
& f_{w_{3}}(x)=\int_{0}^{x} \frac{\theta_{1} \theta_{2}}{\left(\theta_{1}-\theta_{2}\right)}\left[e^{-\theta_{2} x}-e^{-\theta_{1} x}\right] \theta_{3} e^{-\theta_{3}(x-y)} d y \\
& =\frac{\theta_{1} \theta_{2} \theta_{3}}{\left(\theta_{1}-\theta_{2}\right)}\left[e^{-\theta_{2} x}-e^{-\theta_{1} x}\right] \int_{0}^{x} e^{-\theta_{3}(x-y)} d y \\
& =\frac{\theta_{1} \theta_{2} \theta_{3}}{\left(\theta_{1}-\theta_{2}\right)}\left[e^{-x\left(\theta_{3}+\theta_{2}\right)} e^{\theta_{3} x}-e^{-x\left(\theta_{3}+\theta_{2}\right)}-e^{-x\left(\theta_{3}+\theta_{1}\right)} e^{\theta_{3} x}+e^{-x\left(\theta_{3}+\theta_{1}\right)}\right] \\
& =\frac{\theta_{1} \theta_{2} \theta_{3}}{\left(\theta_{1}-\theta_{2}\right)}\left[e^{-\theta_{2} x}-e^{-x\left(\theta_{3}+\theta_{2}\right)}-e^{-\theta_{1} x}+e^{-x\left(\theta_{3}+\theta_{1}\right)}\right] \\
& E(C)=h \int_{0}^{s}[S-X] \frac{\theta_{1} \theta_{2} \theta_{3}}{\left(\theta_{1}-\theta_{2}\right)}\left[e^{-\theta_{2} x}-e^{-x\left(\theta_{3}+\theta_{2}\right)}-e^{-\theta_{1} x}+e^{-x\left(\theta_{3}+\theta_{1}\right)}\right] d x \\
& +d \int_{S}^{\infty}[X-S] \frac{\theta_{1} \theta_{2} \theta_{3}}{\left(\theta_{1}-\theta_{2}\right)}\left[e^{-\theta_{2} x}-e^{-x\left(\theta_{3}+\theta_{2}\right)}-e^{-\theta_{1} x}+e^{-x\left(\theta_{3}+\theta_{1}\right)}\right] d x \\
& =\frac{h \theta_{1} \theta_{2} \theta_{3}}{\left(\theta_{1}-\theta_{2}\right)}\left[\int_{0}^{s}[S-X]\left[e^{-\theta_{2} x}-e^{-x\left(\theta_{3}+\theta_{2}\right)}-e^{-\theta_{1} x}+e^{-x\left(\theta_{3}+\theta_{1}\right)}\right] d x\right] \\
& +\frac{d \theta_{1} \theta_{2} \theta_{3}}{\left(\theta_{1}-\theta_{2}\right)}\left[\int_{s}^{\infty}[X-S]\left[e^{-\theta_{2} x}-e^{-x\left(\theta_{3}+\theta_{2}\right)}-e^{-\theta_{1} x}+e^{-x\left(\theta_{3}+\theta_{1}\right)}\right] d x\right]
\end{aligned}
$$

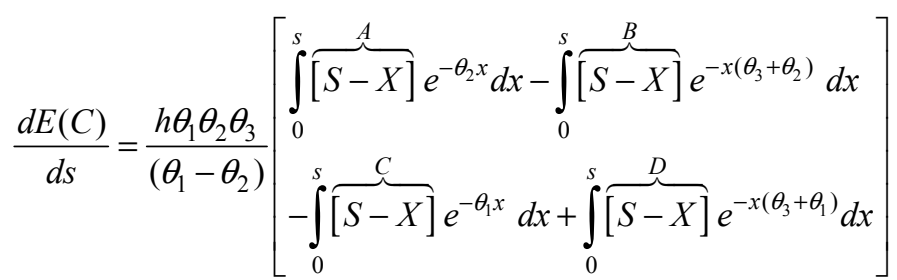

$$
\begin{aligned}
& +\frac{d \theta_{1} \theta_{2} \theta_{3}}{\left(\theta_{1}-\theta_{2}\right)}\left[\begin{array}{l}
\int_{s}^{\infty} \overbrace{[X-S]}^{E} e^{-\theta_{2} x} d x-\int_{s}^{\infty} \overbrace{[X-S]}^{F} e^{-x\left(\theta_{3}+\theta_{2}\right)} d x] \\
-\int_{s}^{\infty} \overbrace{[X-S]}^{G} e^{-\theta_{1} x} d x+\int_{s}^{\infty} \overbrace{[X-S]}^{H} e^{-x\left(\theta_{3}+\theta_{1}\right)} d x]
\end{array}\right\}=0
\end{aligned}
$$




$$
\mathrm{A}=\int_{0}^{s}[S-X] e^{-\theta_{2} x} d x
$$

By Leibenitz rule

$$
\begin{gathered}
\varphi(s)=0 ; \quad \varphi^{1}(s)=0 ; \quad \psi(s)=s ; \psi^{1}(s)=1 ; \\
\frac{d A}{d s}=\frac{1}{\theta_{2}}\left[1-e^{-\theta_{2} s}\right] \\
\mathrm{B}=\int_{0}^{s}[S-X] e^{-x\left(\theta_{3}+\theta_{2}\right)} d x
\end{gathered}
$$

$$
\begin{gathered}
\frac{d B}{d s}=\frac{1}{\left(\theta_{3}+\theta_{2}\right)}\left[1-e^{-s\left(\theta_{3}+\theta_{2}\right)}\right] \\
\mathrm{C}=\int_{0}^{s}[S-X] e^{-\theta_{1} x} d x
\end{gathered}
$$

By Leibenitz rule

$$
\begin{gathered}
\frac{d C}{d s}=\frac{1}{\theta_{1}}\left[1-e^{-\theta_{1} s}\right] \\
\mathrm{D}=\int_{0}^{s}[S-X] e^{-x\left(\theta_{3}+\theta_{1}\right)} d x
\end{gathered}
$$

By Leibenitz rule

$$
\begin{aligned}
& \varphi(s)=0 ; \quad \varphi^{1}(s)=0 ; \quad \psi(s)=s ; \psi^{1}(s)=1 ; \\
& \frac{d D}{d s}=\frac{1}{\left(\theta_{3}+\theta_{1}\right)}\left[1-e^{-s\left(\theta_{3}+\theta_{1}\right)}\right] \\
& \left.\begin{array}{rl}
\frac{d E(C)}{d s} & =\frac{h \theta_{1} \theta_{2} \theta_{3}}{\left(\theta_{1}-\theta_{2}\right)}\left[\frac{1}{\theta_{2}}\left[1-e^{-\theta_{2} s}\right]-\frac{1}{\left(\theta_{3}+\theta_{2}\right)}\left[1-e^{-s\left(\theta_{3}+\theta_{2}\right)}\right]-\frac{1}{\theta_{1}}\left[1-e^{-\theta_{1} s}\right]+\frac{1}{\left(\theta_{3}+\theta_{1}\right)}\left[1-e^{-s\left(\theta_{3}+\theta_{1}\right)}\right]\right] \\
& +\frac{d \theta_{1} \theta_{2} \theta_{3}}{\left(\theta_{1}-\theta_{2}\right)}\left[-\frac{1}{\theta_{2}}\left[e^{-\theta_{2} s}\right]+\frac{1}{\left(\theta_{3}+\theta_{2}\right)}\left[e^{-s\left(\theta_{3}+\theta_{2}\right)}\right]+\frac{1}{\theta_{1}}\left[e^{-\theta_{1} s}\right]-\frac{1}{\left(\theta_{3}+\theta_{1}\right)}\left[e^{-s\left(\theta_{3}+\theta_{1}\right)}\right]\right]
\end{array}\right\}=0 \\
& =\frac{\theta_{1} \theta_{2} \theta_{3}}{\left(\theta_{1}-\theta_{2}\right)}\left[\begin{array}{c}
h\left[\frac{1}{\theta_{2}}\left[1-e^{-\theta_{2} s}\right]-\frac{1}{\left(\theta_{3}+\theta_{2}\right)}\left[1-e^{-s\left(\theta_{3}+\theta_{2}\right)}\right]-\frac{1}{\theta_{1}}\left[1-e^{-\theta_{1} s}\right]+\frac{1}{\left(\theta_{3}+\theta_{1}\right)}\left[1-e^{-s\left(\theta_{3}+\theta_{1}\right)}\right]\right] \\
+d\left[-\frac{1}{\theta_{2}}\left[e^{-\theta_{2} s}\right]+\frac{1}{\left(\theta_{3}+\theta_{2}\right)}\left[e^{-s\left(\theta_{3}+\theta_{2}\right)}\right]+\frac{1}{\theta_{1}}\left[e^{-\theta_{1} s}\right]-\frac{1}{\left(\theta_{3}+\theta_{1}\right)}\left[e^{-s\left(\theta_{3}+\theta_{1}\right)}\right]\right]
\end{array}\right]=0
\end{aligned}
$$

By Leibenitz rule

$$
\begin{gathered}
\frac{d F}{d s}=\frac{-1}{\left(\theta_{3}+\theta_{2}\right)}\left[e^{-s\left(\theta_{3}+\theta_{2}\right)}\right] \\
\mathrm{G}=\int_{s}^{\infty}[X-S] e^{-\theta_{1} x} d x \\
\frac{d G}{d S}=-\frac{1}{\theta_{1}}\left[e^{-\theta_{1} s}\right]
\end{gathered}
$$

By Leibenitz rule

$$
\frac{d H}{d S}=\frac{-1}{\left(\theta_{3}+\theta_{2}\right)}\left[e^{-s\left(\theta_{3}+\theta_{1}\right)}\right]
$$

substituting (9), (10), (11), (12), (13), (14), (15) and (16)$$
\mathrm{H}=\int_{S}^{\infty}[X-S] e^{-x\left(\theta_{3}+\theta_{1}\right)} d x
$$ 


$$
\left[\begin{array}{l}
\frac{h}{\theta_{2}}-\frac{h}{\theta_{2}} e^{-\theta_{2} s}-\frac{h}{\left(\theta_{3}+\theta_{2}\right)}+\frac{h}{\left(\theta_{3}+\theta_{2}\right)} e^{-s\left(\theta_{3}+\theta_{2}\right)}-\frac{h}{\theta_{1}}+\frac{h}{\theta_{1}} e^{-\theta_{1} s}+\frac{h}{\left(\theta_{3}+\theta_{1}\right)}-\frac{h}{\left(\theta_{3}+\theta_{1}\right)} e^{-s\left(\theta_{3}+\theta_{1}\right)} \\
-\frac{d}{\theta_{2}} e^{-\theta_{2} s}+\frac{d}{\left(\theta_{3}+\theta_{2}\right)} e^{-s\left(\theta_{3}+\theta_{2}\right)}+\frac{d}{\theta_{1}} e^{-\theta_{1} s}-\frac{d}{\left(\theta_{3}+\theta_{1}\right)} e^{-s\left(\theta_{3}+\theta_{1}\right)}
\end{array}\right]=0
$$

on simplification we get

$$
\frac{h}{(h+d)}=\left[\begin{array}{l}
\left(\theta_{3}+\theta_{2}\right) \theta_{1}\left(\theta_{3}+\theta_{1}\right) e^{-\theta_{2} s}-\theta_{2} \theta_{1}\left(\theta_{3}+\theta_{1}\right) e^{-s\left(\theta_{3}+\theta_{2}\right)} \\
\frac{-\theta_{2}\left(\theta_{3}+\theta_{2}\right)\left(\theta_{3}+\theta_{1}\right) e^{-\theta_{1} s}+\theta_{2}\left(\theta_{3}+\theta_{2}\right) \theta_{1} e^{-s\left(\theta_{3}+\theta_{1}\right)}}{\theta_{3}^{2} \theta_{1}+\theta_{3} \theta_{1}^{2}-\theta_{3}^{2} \theta_{2}-\theta_{2}^{2} \theta_{3}}
\end{array}\right]
$$

Any value of $\mathrm{S}$ which satisfies equation (17) for specific values

$\theta_{1}, \theta_{2}, \mathrm{~h}$ and $\mathrm{d}$ given the optimal value of $\mathrm{S}$.

$$
\begin{gathered}
\theta_{1}=1.75, \quad \theta_{2}=1.5, \quad \theta_{3}=1.25, \quad h=20, \quad d=120 \\
\frac{20}{140}=\frac{14.4375 e^{-1.5 s}+7.21875 e^{-3 s}-7.875 e^{-2.775 s}-12.375 e^{-1.75 s}}{1.40625} \\
\frac{20}{140} \times 1.40625=14.4375 e^{-1.5 s}+7.21875 e^{-3 s}-7.875 e^{-2.775 s}-12.375 e^{-1.75 s}
\end{gathered}
$$

LHS $=0.2008$

$\mathrm{S}=4$ R. H. $\mathrm{S}=0.2530$ LHS $=0.21$ (convert to two decimal places)

$\mathrm{S}=4.1$ R. H. $\mathrm{S}=0.2297$ LHS $=0.21$ (convert to two decimal places)

$$
\begin{aligned}
& \mathrm{S}=4.2 \rightarrow 0.2084 \\
& \mathrm{~S}=4.24 \rightarrow 0.20054 \\
& \mathrm{RHS}=0.2005 \\
& \hat{S}=4.24
\end{aligned}
$$

Since L. H. S and R. H. S are equal.

\section{Conclusion}

On the basis of the model for finding the optimal supply when demand is a sum of two components as discussed. The conclusions drawn are (1) If the demand due to consumer purchase and demand due to transfer produce to sister companies are both random variables following exponential distributions then as $\mathrm{h}$ increases a higher level of $S$ is suggested. (2) As d the shortage cost increases then a larger size of inventory is suggested. Similarly the results are found to be identical when the demand can be portrayed as the sum of three random variables or segments.

The validity of these models for practical use depends upon the determination of the distribution of the random vbariables involved in the model. The models will be of practical use when the distribution are formulated based on the data collected from practical situations. Also the test for goodness of fit for the distributions will improve the accuracy of the model and the optimal solutions will be with greater precision.

\section{References}

[1] Amanda J. Schmitt, Lawrence V. Snyder, Zuo- Jun Max Shen (2008). Inventory Systems with Stochastic Demand and supply: Properties and Aproximations, http://ssrn.com, pp. 131.

[2] Barber. J. H. (1925). Economic control Inventory, New York Codes Book CO.

[3] Bellman. R. (1956a). On the theory of Dynamic programming - A ware housing problem, Management science, Volume 2, (No. 3): pp. 272-27.

[4] Bellman. R. (1956b). Dynamic programming and the smoothing problems, Management science, Volume 3, (No. 1): pp. 111-113.

[5] Beyer. D and Sethi. S. P. (1997). Average cost optimality in inventory Models with Markovian demands, Journal of Optimization theory and Applications, Vol. 92, No. 3, pp. 497-526.

[6] Bishop. G. R. (1957). On a Problems of production scheduling Operations Research, Vol. 5, (No. 1): pp. 97-103.

[7] Bowman, E. H., Richard D Irwin and Fetter, R. B. (1957). Analysis for production management. Home wood - Illinois.

[8] Brill Percy. H. And Ben A Chaouch (1995). An EOQ model with Random variables in demand-Management science, 41, 5 pp. 927-936.

[9] T. Venkatesan C. Muthu And R. Sathiyamoorthy (2010). Determination of Optimal Reserves between Two Machines in Series. Journal of Ultra Scientist of Physical Sciences, Vol. 22 (3) $M$, (2010), pp 853-861. 
[10] T. Venkatesan C. Muthu And R. Sathiyamoorthy (2012). Determination of Optimal Reserve of Semi Finished products between three machines in series. Journal of Indian Academy of Mathematics, V0l. 34, No. 1 (2012), pp: 175-184.

[11] T. Venkatesan C. Muthu And R. Sathiyamoorthy (2016). Determination of Optimal Reserves between Three Machines in Series. International Journal of Advanced Research in
Mathematics and Applications, Volume: 1 Issue: 1 May, 2016 , ISSN_NO: 2350-028X.

[12] T. Venkatesan1, G. Arivazhagan And C. Muthu (2017). Some Applications of Order Statistics in Inventory Control. IOSR Journal of Mathematics (IOSR-JM) e-ISSN: 2278-5728, pISSN: 2319-765X. Volume 13, Issue 1 Ver. IV (Jan. - Feb. 2017). 